\title{
DYT6 Dystonia Mimicking Adolescent Idiopathic Scoliosis Successfully Treated by Pallidal Stimulation
}

\author{
Chun-Hwei Tai ${ }^{\prime}$ \\ Wang-Tso Lee ${ }^{2}$ \\ Sheng-Hong Tseng ${ }^{3}$ \\ 'Department of Neurology, National \\ Taiwan University Hospital and College of \\ Medicine, Taipei, Taiwan; ${ }^{2}$ Department of \\ Pediatrics, National Taiwan University \\ Hospital, Taipei, Taiwan; ${ }^{3}$ Department of \\ Surgery, National Taiwan University \\ Hospital, Taipei, Taiwan
}

Purpose: Dystonia type 6 (DYT6) is an autosomal dominant monogenic movement disorder that often involves craniocervical and laryngeal regions, but can in rare circumstance present as trunk dystonia or severe scoliosis. Deep brain stimulation of the globus pallidus internus (GPi-DBS) has yielded favorable results in the treatment of DYT6 patients. This report describes the case of a 14-year-old male adolescent with DYT6 dystonia and severe scoliosis who was successfully treated by GPi DBS.

Patients and Methods: The diagnosis of DYT6 dystonia was made after excluding other etiologies and was confirmed by next-generation sequencing. The patient underwent bilateral GPi-DBS implantation surgery under general anesthesia.

Results: The patient's Burke-Fahn-Marsden Dystonia Rating Scale score was 24 before surgery and decreased to 13.5 at 3 months, 3 at 6 months, and 2 at 12 months after bilateral GPi-DBS, corresponding to a $91 \%$ improvement from baseline to 12 months post-surgery. The patient's scoliosis improved significantly within 6 months after DBS. No complications occurred during surgery.

Conclusion: An adolescent DYT6 patient with dystonia-related severe scoliosis was treated by bilateral GPi-DBS. The patient had an excellent outcome at 12 months after surgery, which prevented him from developing severe spinal deformity and disability. Early diagnosis of dystonia in adolescent patients can lead to timely and effective treatment. The etiology of severe scoliosis in adolescents should be carefully evaluated and differential diagnosis including dystonia should be considered. GPi-DBS in patients with DYT6 dystonia can prevent deformity.

Keywords: dystonia type 6, movement disorder, spinal deformity, deep brain stimulation, globus pallidus internus

\section{Introduction}

Correspondence: Sheng-Hong Tseng National Taiwan University Hospital, No. 7, Zhong-Shan South Road, Taipei, I00, Taiwan

Tel $+88622312-3456$ ext.65II0

Fax +886 2234I-8395

Email tsh5110@ntu.edu.tw

Wang-Tso Lee

National Taiwan University Hospital, No.

7, Zhong-Shan South Road, Taipei, I00,

Taiwan

Tel +886 223|2-3456 ext.7|5|4

$\mathrm{Fax}+88622314-7450$

Email leeped@hotmail.com
Dystonia type 6 (DYT6) is an autosomal dominant monogenic isolated movement disorder caused by mutations in the thanatos-associated domain-containing apoptosis-associated protein (THAP) 1 gene. ${ }^{1}$ Clinically, DYT6 is characterized by an early age of onset - usually during childhood or adolescence-with symptoms that frequently start in the craniocervical region (46\%), extremities (44\%), or laryngeal region $(10 \%))^{1,2}$ Speech involvement is also common. ${ }^{2,3}$ Dystonia symptoms often develop progressively and can result in severe disability. Severe trunk dystonia as the main presenting symptom is relatively uncommon; ${ }^{2,4}$ in adolescent patients, this can sometimes mimic idiopathic adolescent scoliosis. ${ }^{5}$ In fact, the cause of scoliosis 
in children and adolescents may be idiopathic (up to $80 \%$ of cases), congenital, or neuromuscular; ${ }^{6}$ neuromuscular etiology as in DYT6 dystonia is a rare but important differential diagnosis in these patients. ${ }^{7}$

With the increasing availability of new genetic diagnostic tools such as next-generation sequencing, it is possible to perform genetic screening tests in adolescent patients with suspected dystonia symptoms even when there is no family history. ${ }^{5}$ Early identification of a genetic etiology for dystonia is critical because a correct diagnosis can ensure timely and appropriate treatment such as deep brain stimulation (DBS) before disability or deformity occurs. ${ }^{8}$ DBS of the globus pallidus internus (GPi) is an effective treatment for primary dystonia with a genetic cause such as DYT1 and DYT6. ${ }^{8,9}$ However, because of the invasiveness and high cost, few patients with DYT6 dystonia have undergone DBS treatment. An earlier small case series reported only moderate responses to DBS. ${ }^{10}$ However, in recent studies, more medically refractory DYT6 cases receiving GPi-DBS showed good outcome, and in DYT6 patients with trunk dystonia, the overall benefit was reported to be comparable to that in other primary dystonias, with a greater effect observed in the trunk and limbs than in the craniocervical region. ${ }^{4,10,11}$

Here, we report the case of a 14-year-old boy with DYT6 dystonia in whom the initial symptom was mild hand clumsiness, followed by rapid progression to debilitating scoliosis. Although it was once considered as idiopathic adolescent scoliosis, the scoliosis progressed despite various treatments. Thanatos-associated domaincontaining apoptosis-associated protein (THAP)1 mutation was confirmed after a diagnostic workup by a pediatric neurologist. Based on suspicion of DYT6 with severe trunk dystonia, the patient received bilateral GPi-DBS treatment. The DYT6-induced scoliosis improved significantly, and disability and deformity were avoided.

\section{Case Description}

\section{History}

The 14-year-old male patient had normal birth and developmental milestones. He has no family history of dystonia or other movement disorders. At the age of 7, the first symptom of dystonia appeared as clumsiness of the hands; when he started to attend elementary school, he experienced difficulty with schoolwork because of an abnormal handgrip during writing. He was also noted to have difficulty playing with small objects such as building blocks or certain musical instruments such as a flute or piano. At first, the condition progressed very slowly. At the age of 10, the patient's father became aware that his son's hand clumsiness was not correlated with his age and development, and sought medical help. The boy visited a rehabilitation clinic for the hand clumsiness and underwent a series of rehabilitation treatments without obvious improvement. He also visited a pediatric neurologist for evaluation, but the brain magnetic resonance imaging (MRI) examination revealed only a parapineal arachnoid cyst, and no specific diagnosis was made at that time. However, when the patient was 11 years old, scoliosis appeared that mainly involved the thoracic and lumbar regions of the spine and abnormal posture, which rapidly deteriorated in the following 6 months. His gait and stance became severely impeded due to the severity of the scoliosis. He visited orthopedic doctors at the local hospital and received various medications, botulinum toxin injection, a back brace, and different types of physical therapy based on an initial suspicion of adult idiopathic scoliosis. None of these treatments were effective for the scoliosis. Muscle biopsy was performed but the results were normal. He was finally referred to a pediatric neurologist (WTL) at our center. Levodopa was given as a trial but had no effect. Laboratory biochemical tests showed no abnormalities and medication-induced and acquired dystonia were excluded. Genetic testing with a dystonia panel by nextgeneration sequencing revealed a heterozygous mutation in the THAP1 gene (c.537dupA).

A neurologic examination showed that the patient had normal cognitive function. His muscle strength was normal except for mild weakness in both hands. Mild bradykinesia of both hands was noted when the patient was asked to perform rapid, alternating movements. He had markedly abnormal posture caused by dystonia involving the spine from the cervical to lumbar segments that prevented him from walking and sitting normally, but his supine posture was nearly normal. The spine plain film showed a Cobb angle of $45^{\circ}$. Given that the scoliosis was progressive and severe, intractable to all treatment measures, and interfered with the patient's daily life and school activities, bilateral GPi-DBS for generalized dystonia was recommended as the treatment when the patient was 12 years old. 


\section{Surgery}

Bilateral GPi-DBS was performed after proper evaluation. The patient underwent a brain MRI scan under general anesthesia, and the images were used for surgical planning with the StealthStation S7 surgical navigation system (Medtronic, Minneapolis, MN, USA), with trajectories targeting bilateral GPi. Frameless stereotaxy with the NexFrame system (Medtronic) under image-guided navigation was carried out on the second day of surgical planning. The surgery was performed under general anesthesia (Figure 1A). Intraoperative electrophysiologic recordings on both sides showed single-unit activity in the GPi, and the trajectory to the optic tract was verified by photic stimulation tests. The patient had no abnormal involuntary movements or contractions during test stimulation. Bilateral DBS electrodes were implanted and confirmed on intraoperative computed tomography $\mathrm{O}$-arm images (Medtronic) immediately after fusion with the preoperatively planned trajectory (Figure 1B-D). The electrodes were connected through extension cables to the implantable pulse generator during the second stage of the operation. There were no surgery-related complications.

\section{Post-Surgery Follow-Up}

GPi-DBS treatment was initiated 1 week after surgery. After testing the therapeutic window of each individual electrode, the parameters were set as follows: left GPi $0(-)$ $\mathrm{C}(+) 1.0 \mathrm{mAmp}, 450 \mu \mathrm{s}, 130 \mathrm{~Hz}$; and right GPi 8(-) $\mathrm{C}(+)$ $1.0 \mathrm{mAmp}, 450 \mu \mathrm{s}, 130 \mathrm{~Hz}$. No stimulation-induced side effects were noted. Zonisamide was continuously administered after DBS. The patient's Burke-Fahn-Marsden Dystonia Rating Scale score was 24 before DBS, and $13.5,3$, and 2 at 3,6 , and 12 months after DBS, respectively. There was a $91 \%$ improvement in the score from baseline (pre-operation) to 12 months after DBS (Figure 2A). The patient's scoliosis improved significantly within first 6 months after the surgery, with the Cobb angle decreasing from $45^{\circ}$ preoperatively to $20^{\circ}$ at the 12 month follow-up (Figure 2B). Only mild cervical and trunk dystonia persisted. At 12 months after DBS, the patient returned to a relatively normal life at home and at school, and was able to performed many physical activities, such as running, jumping, and cycling with the improvement of his scoliosis (Figure 2C). The rehabilitation program was continued for twelve months to further improve posture and fine motor movements.

\section{Discussion}

We performed bilateral GPi-DBS in a 12-year-old boy with DYT6 (THAP1 mutation) presenting with severe scoliosis. There was a significant and lasting improvement of the DYT6 dystonia-related scoliosis at the 1-year follow-up, and the patient was able to return to a relatively normal family and school life.

This case demonstrates that early diagnosis of genetic dystonia in childhood or adolescence is essential as it may be treatable with the latest medical technology (ie, GPi-DBS) .$^{8,9}$ The initial diagnostic workup of these patients is often challenging, and many are diagnosed at a late stage of disease if the dystonia is not correctly identified or if genetic testing tools are not readily available. In our case, the onset of severe, debilitating scoliosis had masked the earlier onset of hand clumsiness, which turned out to be an important clue for the diagnosis of dystonia. ${ }^{13}$ In fact, identifying dystonia symptomology is the key first step to a correct diagnosis. A new diagnostic algorithm for dystonia in children and adolescents has been proposed that includes identifying dystonia, screening for medication/toxin-induced or acquired dystonia, biochemical testing and levodopa trial, and next-generation sequencing. ${ }^{5}$ Investigating dystonia etiologies in this manner is useful for the early diagnosis of dystonia in adolescents, as was the case in our patient.

Scoliosis is a relatively common orthopedic condition in children and adolescents. ${ }^{6}$ A neuromuscular cause is rare but important to determine, as identification of dystonia may influence diagnosis and treatment decisions. ${ }^{4,7}$ In adolescent patients with dystonia, the scoliosis curve magnitude often increases rapidly with skeletal growth. Additionally, skeletally immature patients with curves $>20^{\circ}$ are high risk for progression. ${ }^{6}$ Our patient experienced scoliosis onset at the age of 10 years with a curvature $>30^{\circ}$, indicating a high risk of rapid scoliosis progression. This further emphasizes the importance of diagnosing dystonia as early as possible in adolescent patients in order to prevent deformities and disabilities. $^{13}$

Predictors of good outcome following GPi-DBS for generalized dystonia include primary (with a genetic cause such as DYT1 or DYT6 mutation) or mobile dystonia, short onset duration, and younger age when undergoing DBS. ${ }^{14,15}$ In our case, a genetic diagnosis was confirmed (DYT6 gene mutation); moreover, the patient had mobile dystonia (no fixed dystonia noted during rest or sleep), a short interval between the onset of dystonia and DBS surgery (1-2 years), and younger age (12 years old), all of which predicted a positive 


\section{A}

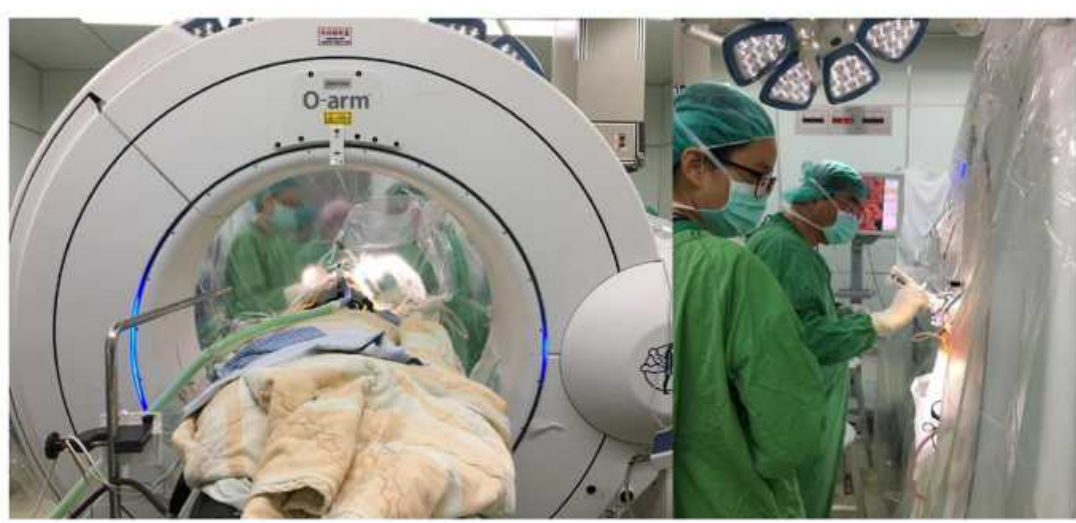

B

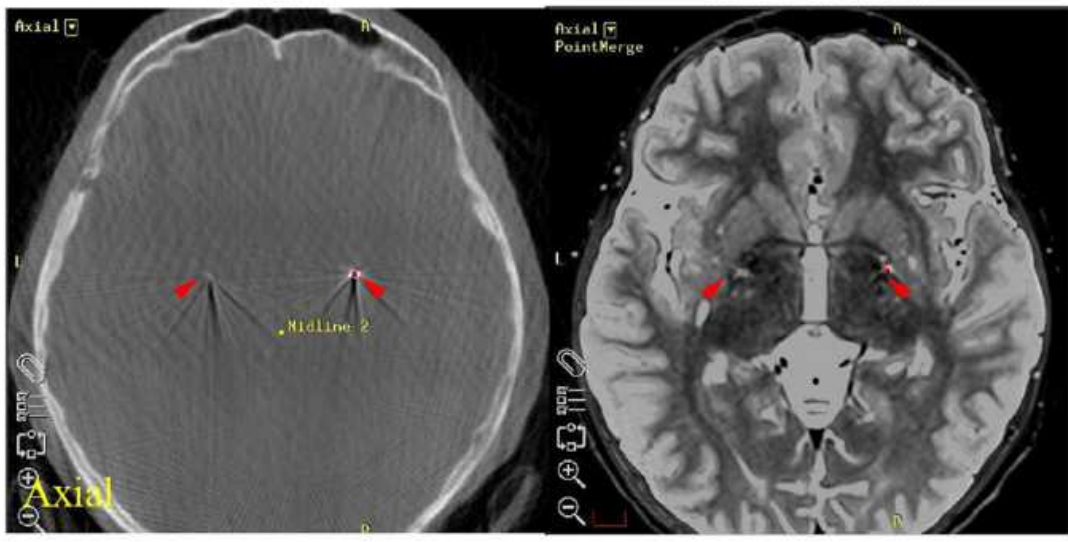

C

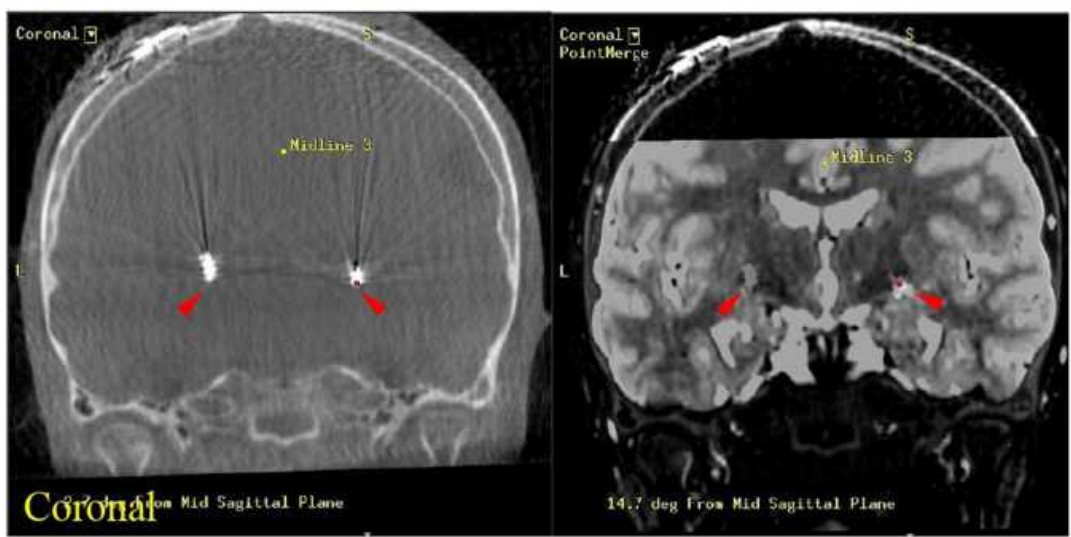

D

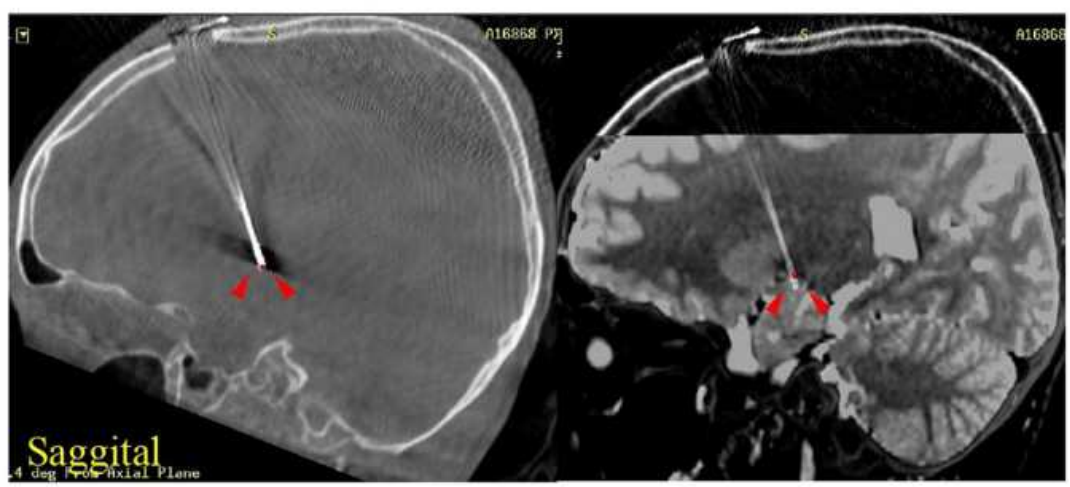

Figure I Bilateral GPi-DBS in an adolescent DYT6 dystonia patient. (A) Image-guided frameless stereotaxic surgery was performed to implant DBS leads into GPi in the I2year-old boy under general anesthesia. (B) Axial, (C) coronal and (D) sagittal images of O-arm (left panel, arrowheads), and fusion images with pre-operative MRI (right panel, arrows) indicating the location of DBS electrodes in bilateral GPi.

Abbreviations: GPi, globus pallidus internus; MRI, magnetic resonance imaging; DBS, deep brain stimulation. 
A

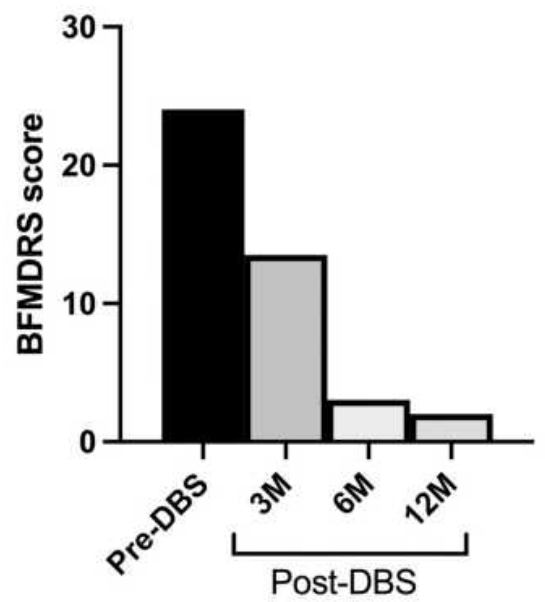

B
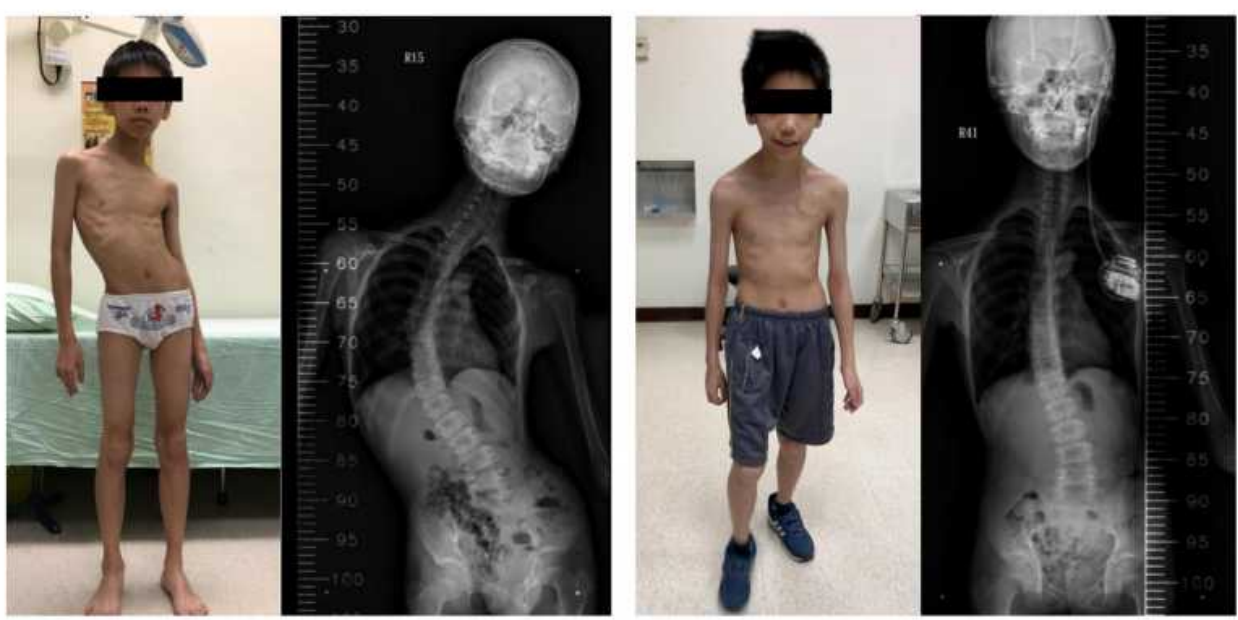

C

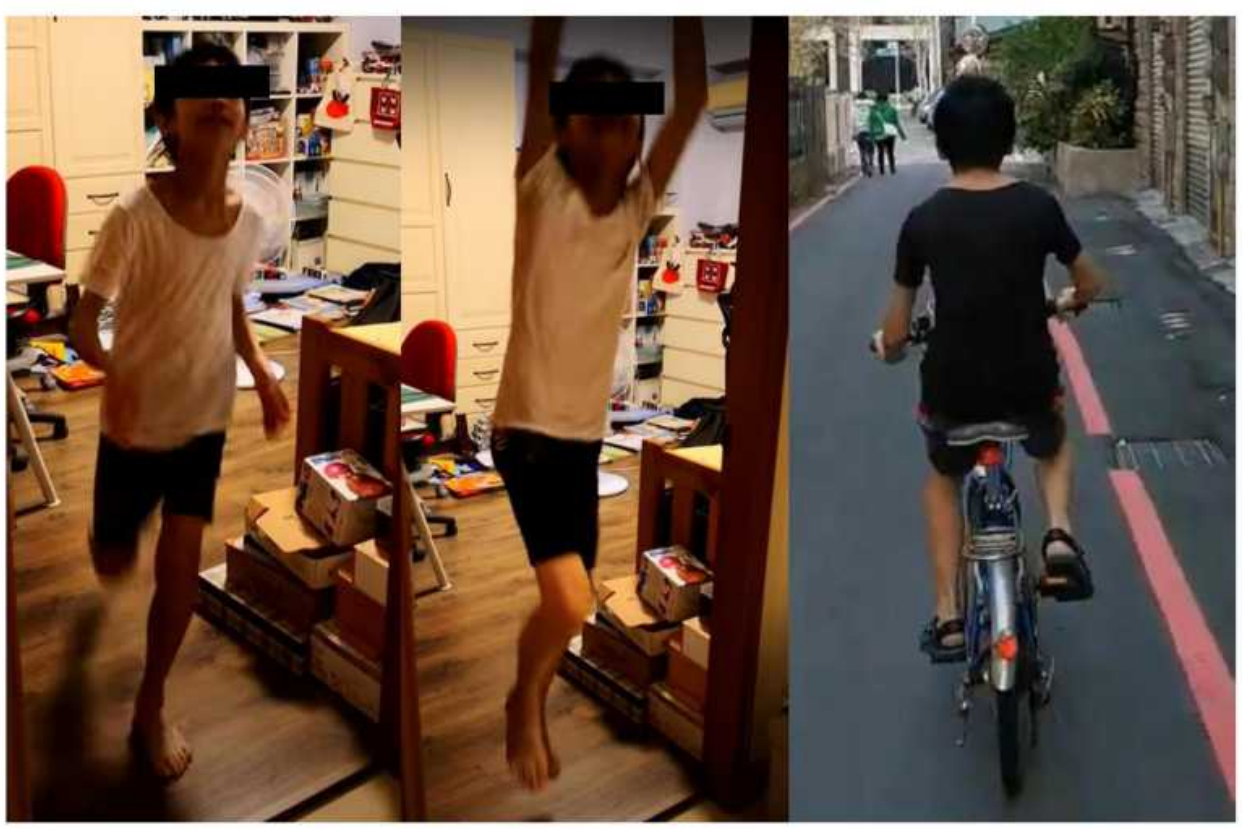

Figure 2 The clinical improvement of the DYT6 dystonia patient after effective bilateral pallidal stimulation. (A) The BFMDRS score changes at baseline and 3-, 6-, and I2months after GPi-DBS. (B) Significant improvement in severe scoliosis of the patient and findings of spine x-rays pre-operatively and at 6-months after GPi-DBS. (C) Photos showing his daily physical activity nearly normal state after successful treatment of scoliosis and dystonia at 12 months after GPi-DBS. 
outcome after GPi-DBS. A previous case series showed that while improvement is to be expected after GPi-DBS for dystonia, it is less robust and predictable for DYT6 than for DYT1. ${ }^{10}$ A possible reason for this is that DYT6 patients often exhibit prominent craniocervical or oropharyngeal dystonia, which is less responsive to DBS targeting the $\mathrm{GPi}^{4,11}$ than dystonia affecting the trunk and limbs. ${ }^{12}$ Other targets such as the ventral oralis anterior nucleus of the thalamus may be evaluated in the future treatment of DYT6 patients with craniocervical or oropharyngeal dystonia as the major presenting feature. ${ }^{16}$ In general, DBS is a safe and well-tolerated procedure for pediatric patients.

In adolescent patients with progressive and severe scoliosis, treatable neuromuscular causes should always be sought. Early identification of dystonia symptoms followed by a diagnostic algorithm that includes appropriate genetic screening can lead to correct diagnosis and early treatment. Our case provides a clear illustration of this concept. GPi-DBS is highly effective in the treatment of primary generalized dystonia, which was the case in our DYT6 patient. We suggest that GPiDBS should always be considered in DYT6 patients with dystonia affecting either the trunk or limbs. DYT6 has rarely been reported in Asian countries; this rare dystonia case with uncommon presentation is a reminder to clinicians in the AsiaPacific region to be aware of dystonia patients with treatable etiology.

In conclusion, DYT6 should be considered as an important etiology of generalized dystonia in adolescent patients; early diagnosis of this condition is important, as GPi-DBS is relatively safe and highly effective in preventing spinal deformity and disability.

\section{Abbreviations}

DBS, deep brain stimulation; DYT6, dystonia type 6; GPi, globus pallidus internus; MRI, magnetic resonance imaging; THAP1, thanatos-associated domain-containing apoptosis-associated protein 1 .

\section{Data Sharing Statement}

Data in this article are available from the corresponding author upon request.

\section{Ethics Approval and Informed Consent}

Written, informed consent was provided by the patient and his parents to have the case details and images published.
Institutional approval was not required to publish case details.

\section{Acknowledgments}

We would like to thank Jill Lin and Joan Lee for assistance in data collection. We would also like to thank Alice Hsu for manuscript preparation.

\section{Funding}

There is no funding to report.

\section{Disclosure}

The authors have no conflicts of interest to declare.

\section{References}

1. Fuchs T, Gavarini S, Saunders-Pullman R, et al. Mutations in the THAP1 gene are responsible for DYT6 primary torsion dystonia. Nat Genet. 2009;41:286-288. doi:10.1038/ng.304

2. Xiromerisiou G, Houlden H, Scarmeas N, et al. THAP1 mutations and dystonia phenotypes: genotype phenotype correlations. Mov Disord. 2012;27:1290-1294. doi:10.1002/mds.25146

3. Djarmati A, Schneider SA, Lohmann K, et al. Mutations in THAP1 (DYT6) and generalized dystonia with prominent spasmodic dysphonia: a genetic screening study. Lancet Neurol. 2009;8:447-452. doi:10.1016/S1474-4422(09)70083-3

4. Krause P, Brüggemann N, Völzmann S, et al. Long-term effect on dystonia after pallidal deep brain stimulation (DBS) in three members of a family with a THAP1 mutation. $J$ Neurol. 2015;262:2739-2744. doi:10.1007/s00415-015-7908-z

5. van Egmond ME, Kuiper A, Eggink H, et al. Dystonia in children and adolescents: a systematic review and a new diagnostic algorithm. J Neurol Neurosurg Psychiatry. 2015;86:774-871. doi:10.1136/jnnp2014-309106

6. El-Hawary R, Chukwunyerenwa C. Update on evaluation and treatment of scoliosis. Pediatr Clin North Am. 2014;61:1223-1241. doi:10.1016/j.pcl.2014.08.007

7. Horne JP, Flannery R, Usman S. Adolescent idiopathic scoliosis: diagnosis and management. Am Fam Physician. 2014;1(89):193-198.

8. Holloway KL, Baron MS, Brown R, Cifu DX, Carne W, Ramakrishnan V. Deep brain for dystonia: a meta-analysis. Neuromodulation. 2006;9:253-261. doi:10.1111/j.1525-1403.2006.00067.x

9. Vidailhet M, Vercueil L, Houeto JL, et al. Bilateral, pallidal, deep-brain stimulation in primary generalised dystonia: a prospective 3 year follow-up study. Lancet Neurol. 2007;6:223-229. doi:10.1016/S1474-4422(07)70035-2

10. Panov F, Tagliati M, Ozelius LJ, et al. Pallidal deep brain stimulation for DYT6 dystonia. $J$ Neurol Neurosurg Psychiatry. 2012;83:182-187. doi:10.1136/jnnp-2011-300979

11. Danielsson A, Carecchio M, Cif L, et al. Pallidal deep brain stimulation in DYT6 dystonia: clinical outcome and predictive factors for motor improvement. J Clin Med. 2019;8:2163. doi:10.3390/jcm8122163

12. Vuletic V, Chudy D, Almahariq F, Dobricic V, Kostic V, Bogdanovic N. Excellent outcome of pallidal deep brain stimulation in DYT6 dystonia: a case report. J Neurol Sci. 2016;366:18-19. doi:10.1016/j.jns.2016.04.032

13. Lin JP, Lumsden DE, Gimeno H, Kaminska M. The impact and prognosis for dystonia in childhood including dystonic cerebral palsy: a clinical and demographic tertiary cohort study. J Neurol Neurosurg Psychiatry. 2014;85:1239-1244. doi:10.1136/jnnp-2013307041 
14. Bronte-Stewart H, Taira T, Valldeoriola F, et al. Inclusion and exclusion criteria for DBS in dystonia. Mov Disord. 2011;26:S5-S16. doi: $10.1002 / \mathrm{mds} .23482$

15. Moro E, LeReun C, Krauss JK, et al. Efficacy of pallidal stimulation in isolated dystonia: a systematic review and meta-analysis. Eur J Neurol. 2017;24:552-560. doi:10.1111/ene.13255
16. Taira T, Horisawa S, Takeda N, Ghate P. Stereotactic radiofrequency lesioning for movement disorders. Prog Neurol Surg. 2018;33:107-119.

\section{Publish your work in this journal}

The International Medical Case Reports Journal is an international, peer-reviewed open-access journal publishing original case reports from all medical specialties. Previously unpublished medical posters are also accepted relating to any area of clinical or preclinica science. Submissions should not normally exceed 2,000 words or 4 published pages including figures, diagrams and references. The manuscript management system is completely online and includes a very quick and fair peer-review system, which is all easy to use. Visit http://www.dovepress.com/testimonials.php to read real quotes from published authors. 